elastography-USG scores were shown to be higher in patients with sialometry of $\leq 1,5 \mathrm{ml}(n=7)(28 \pm 3$ vs $17 \pm 10, p=0,010,8 \pm 1$ vs $5 \pm 3, p=0006$ and $9 \pm 1$ vs $5 \pm 2$, $p=0,028)$ and anti-Ro positivity $(n=24)(24 \pm 10$ vs $13 \pm 87 \pm 3$ vs $4 \pm 2, p<0001$ and 7 \pm 2 vs $3 \pm 2, p=0,003$ ). The patients with severe parotid involvement (inhomogeneity/hypoechogenic areals $\geq 2$ ) had more frequent anti-Ro and anti-La positivity (80 vs $42 \%, p=0004$ and 48 vs $17 \%, p=0,011$ )

Abstract THU0372 - Table 1. Demographics and Clinical Characteristics of SjS patients.

\begin{tabular}{|l|c|}
\hline & $\mathrm{n}=75$ \\
\hline Sicca symptoms & $69(92 \%)$ \\
\hline Arthralgia/arthritis & $62(83 \%)$ \\
\hline Parotitis & $20(27 \%)$ \\
\hline Raynaud Phenomenon & $12(16 \%)$ \\
\hline Leucocytoclastic vasculitis & $5(7 \%)$ \\
\hline Peripheral neuropathy & $7(9 \%)$ \\
\hline Interstitial lung disease & $3(4 \%)$ \\
\hline Lymphadenopathy & $12(16 \%)$ \\
\hline Congenital heart block & $2(3 \%)$ \\
\hline ANA & $62(82 \%)$ \\
\hline Anti-Ro/La & $35(47 \%)$ \\
\hline ESR(mm/h) & $32 \pm 20$ \\
\hline CRP(mg/l) & $4,8 \pm 6,1$ \\
\hline
\end{tabular}

Abstract THU0372 - Table 2. Disease Activity Indexes and SG-USG Scores of SjS Patients

\begin{tabular}{|l|c|}
\hline & $n=75$ \\
\hline ESSPRI-total & $14,8 \pm 6,5$ \\
\hline -dryness & $5,4 \pm 2,5$ \\
\hline -fatigue & $4,8 \pm 2,8$ \\
\hline -pain & $4,6 \pm 3$ \\
\hline VAS ESSDAl-total & $50 \pm 22$ \\
\hline Hocevar-USG Score & $2,1 \pm 3,5$ \\
\hline Milic-USG Score & $19 \pm 5$ \\
\hline Sialometry (ml) (n=45) & $6 \pm 3$ \\
\hline Elastography ( $n=32)$ & $6,4 \pm 5$ \\
\hline
\end{tabular}

Conclusions: Hocevar scoring system of major salivary glands was found to be related to patient reported activity in SjS. USG scores were associated with reduced saliva secretion and anti-Ro positivity. Severe parotid involvement was shown to be related to anti-RO and La positivity. Evaluation of SG-USG including different scoring systems and elastography might reflect function of the salivary glands.

Disclosure of Interest: None declared

DOI: 10.1136/annrheumdis-2018-eular.7099

\section{THU0373 THE MARKERS USEFUL IN PREDICTING LUPUS NEPHRITISIN CLINICAL PRACTICE}

${ }^{1}$ P. Dabrowski, K. Marczak ${ }^{1}$, A. Gala-Bladzińska ${ }^{2,3}$, K. Gargasz ${ }^{4}$, M. Dabrowska ${ }^{5}$. ${ }^{1}$ Department of Rheumatology, Clinical Provincial Hospital No. 2, Lwowska 60 Street, 35-301 Rzeszów, Poland; ${ }^{2}$ Faculty of Medicine, University of Rzeszów, Kopisto 2a Avenue, 35-359 Rzeszów, Poland; ${ }^{3}$ Dialysis Center, Clinical Provincial Hospital No. 2, Lwowska 60 Street, 35-301 Rzeszów, Poland; ${ }^{4}$ Data Analysis Laboratory, Centre for Innovative Research in Medical and Natural Sciences, Faculty of Medicine, University of Rzeszow, Kopisto 2a Avenue, 35-359 Rzeszów, Poland; ${ }^{5}$ II Department of Pediatrics, Endocrinology and Diabetology, Clinical Provincial Hospital No. 2, Lwowska 60 Street, 35-301, Rzeszów, Poland

Background: Lupus nephritis (LN) is one of the most severe clinical manifestations of systemic lupus erythematosus (SLE). LN can be found in approximately $50 \%$ of SLE patients. The renal biopsy remains the gold diagnostic standard. However non-invasive and clinically practical laboratory markers of kidney damage in this disease are sought.

Objectives: The aim of the study was to assess the utility of biomarkers like inflammable indicators, complement system components and albuminuria in a single urine sample for prediction of kidney involvement and disease activity in patients with SLE.

Methods: A prospective study included 33 patients (81.8\% women) with SLE criteria according to SLICC (Systemic Lupus International Collaborating Clinics) in age of $39.0 \pm 14.3$ years. Disease-duration ranged $6-60$ months. We performed full physician examination and we excluded patients with active infection. The disease activity based on the SELENA-SLEDAI scale was assessed and divided into groups: low activity $(L)<6$, moderate $(M) 7-12$, high $(H)>12$ points.

In the blood samples complement components (C3, C4) (g/L), C-reactive protein CRP $(\mathrm{mg} / \mathrm{L})$, interleukin $6(\mathrm{IL}-6)(\mathrm{pg} / \mathrm{mL})$, serum creatinine concentration $(\mathrm{sCr})$ $(\mathrm{mg} / \mathrm{g})$, ESR $(\mathrm{mm} / \mathrm{h})$, glomerulal filtration rate (eGFR) according to CKD-EPI $(\mathrm{ml} /$ $\mathrm{min} / 1.73 \mathrm{~m}^{2}$ ) were determined. The concentration of albumin (uAlb) (mg/dl) and creatinine $\mathrm{sCr}(\mathrm{g} / \mathrm{dL})$ from the morning urine sample was measured and the albu $\mathrm{min} /$ creatinine index (UACR) $(\mathrm{mg} / \mathrm{g}$ ) was calculated. Based on the obtained results, patients were divided into stages of chronic kidney disease (CKD) according to .KDIGO 2012. A daily proteinuria (DP) (g/24 hour) was performed. In the assessment of statistical significance, Kruskal-Wallis or Mann-Whitney U-tests were used.

Results: In our study the SLE activity was as follows (\%): L-24 (72.7), M-6 (18.2), $\mathrm{H}-3$ (9.1). The average values (range) of biomarkers of renal function were: $\mathrm{Cr}=0,81 \pm 0,27(0.55-1.65), \mathrm{eGFR}=99.6 \pm 24.4(46-131), \mathrm{uAlb}=13.6 \pm 34.4(0.04$ 161.0), uACR=121.3 \pm 356.3 (4.8-1905.3), $D P=0.32 \pm 0.92(0.015-5.3)$, and other biomarkers: $\mathrm{OB}=26.1 \pm 25.9$ (4.0-99.0); $\mathrm{CRP}=10.3 \pm 28.1 \quad(0.2-148.7) ; \mathrm{IL}-6=7.8$ \pm 14.7 (0-78.3); C3=1.08 $\pm 0.36(0.33-2.25)$; C4=0.16 $\pm 0.09(0.02-0.43)$. The study group met the CKD criteria: G1 $n=21(63.6 \%), G 2 n=9(27.3 \%), G 3 n=3(9.1 \%)$ $A 1 n=26(78.8 \%), A 2 n=3(9.1 \%), A 3 n=4(12.1 \%)$.

We showed a negative relationship between the eGFR and: CRP ( $R=-0.49$ $\mathrm{p}=0.005)$, IL-6 $(\mathrm{R}=-0.48, \mathrm{p}=0.005)$ and $\mathrm{C} 4(\mathrm{R}=-0.43, \mathrm{p}=0.01)$. There was also a significant dependence of the SLEDAI SLE activity with: $u A l b(L, H)(p=0.04)$, DP $(\mathrm{M}, \mathrm{H})(\mathrm{p}=0.03), \mathrm{uACR}$ in the whole study group $(\mathrm{p}=0.04)$ and between $\mathrm{ACCR}$ and DP $(p=0.0003)$.

Conclusions: Our studies showed that the risk of kidney damage in SLE may depend on the concentration of CRP, IL-6, C4. In addition albuminuria (UAlb, UACR) correlates with the value of DP and SLE activity, what indicates the dominant glomerular lesion in the etiopathogenesis of proteinuria in $\mathrm{LN}$.

Disclosure of Interest: None declared

DOI: 10.1136/annrheumdis-2018-eular.5867

\section{THU0374 FACTORS ASSOCIATED WITH HIGH-DOSE CORTICOSTEROID USE IN SLE PATIENTS POST INITIATION OF SLE THERAPY}

K. Schroeder ${ }^{1}$, J. Paik ${ }^{1},{ }^{1}$ R.W. Hoffman, S. Gelwicks ${ }^{2} .{ }^{1}$ Eli Lilly and Company; ${ }^{2}$ Former Eli Lilly and Company Employee, Indianapolis, USA

Background: Systemic lupus erythematosus (SLE) therapies include non-steroidal anti-inflammatory drugs, antimalarials, systemic immunosuppressants, and biologics with corticosteroids as necessary. The majority of these current therapies are only partially effective in disease control. Despite treatment, patients may experience flares of disease activity, which can lead to progressive end-organ damage. Severe flares may require intensive immunosuppression, including with high-dose corticosteroids, with risk including end-organ damage.

Objectives: To understand the unmet need in SLE by quantifying use of highdose ( $\geq 40 \mathrm{mg} /$ day) corticosteroids and determining factors associated with its use.

Methods: This study utilised the Truven Marketscan commercial claims database. Patients were indexed on first use of antimalarial, oral immunosuppressant or biologic during 2012-2013 (first use determined based on no claims for the 3 drug classes during the 1 year pre-index). Included patients had 2 recorded SLE diagnoses, were 18-50 years of age and had continuous medical and prescription enrollment from baseline through the 2 year follow-up. Patients with other prespecified autoimmune disorders or cancers during the study period (baseline through follow-up) were excluded. During follow-up, fill of at least 1 high-dose corticosteroid prescription was assessed and associative logistic regression modelling performed.

Results: 1401 patients (93\% female; mean age 38.4 years) met the study criteria; $79 \%$ were indexed on an antimalarial, $15 \%$ on an oral immunosuppressive, $1 \%$ on a biologic and $5 \%$ on a combination of at least 2 of the aforementioned classes. $16 \%$ patients received a diagnosis code for nephritis or chronic kidney disease (CKD), $3 \%$ for myocarditis or pericarditis, and $13 \%$ for thrombocytopenia or leukopenia. During baseline, $56 \%$ of patients had at least 1 visit to a rheumatologist and $13 \%$ filled at least 1 high-dose corticosteroid prescription. During follow-up, $22 \%$ of patients had at least 1 high-dose corticosteroid prescription. Factors significantly associated $(p<0.05)$ with high-dose corticosteroids during follow-up included: baseline rheumatologist visit ( $\mathrm{OR}=0.62 ; 95 \% \mathrm{Cl}=0.47-0.82)$, number of SLE medication classes received during follow-up $(\mathrm{OR}=1.85,95 \% \mathrm{Cl}=1.36$ $2.51)$, receipt of high dose corticosteroid during baseline $(\mathrm{OR}=5.21,95 \%$ $\mathrm{Cl}=3.60-7.53)$, nephritis or CKD $(\mathrm{OR}=1.85,95 \% \mathrm{Cl}=1.29-2.64)$, myocarditis/pericarditis (OR=3.38, 95\% Cl=1.75-6.55), and thrombocytopenia/leukopenia $(\mathrm{OR}=1.70,95 \% \mathrm{Cl}=1.17-2.48)$.

Conclusions: A number of baseline factors were associated with high-dose corticosteroid treatment during the follow-up period; one notable factor is the high percentage of patients using high-dose corticosteroids ( $\geq 40 \mathrm{mg} /$ day). This indicates 